\title{
Abuso y contraindicaciones en el uso de antiinflamatorios no esteroideos
}

\author{
Jesús Rivera-Aguirre ${ }^{1,2 *}$ \\ ${ }^{1}$ Instituto de Ciencias Biomédicas, Universidad Autónoma de Ciudad Juárez, Ciudad Juárez, Chihuahua; '2Unidad de Investigación Traslacional, \\ Fundación Clínica Médica Sur, Ciudad de México. México
}

\begin{abstract}
Resumen
Los antiinflamatorios no esteroideos (AINE) son de los fármacos más ampliamente utilizados en todo el mundo por sus efectos analgésicos antipiréticos y antiinflamatorios, además de otras indicaciones como antiagregantes plaquetarios. En este texto como objetivo se expone una revisión de los efectos adversos más comunes relacionados con el uso de AINE. La automedicación es una práctica frecuente y con múltiples efectos adversos, como úlceras, infección, sangrado y perforación intestinal, hepatotoxicidad e insuficiencia renal aguda, entre otros. La elaboración de esta revisión se realizó en una amplia búsqueda en Science Direct sobre la evidencia más recientemente publicada, además de bibliografías con fundamentos relevantes para este texto, donde se concluye que los efectos adversos representan un problema de salud pública al aumentar el índice de mortalidad en las salas de emergencia de todo el país.
\end{abstract}

Palabras clave: Farmaco-terapéutica. Toxicidad. Patología.

\section{Abuse and contraindications of non-steroidal anti-inflammatory drugs}

\section{Abstract}

Nonsteroidal anti-inflammatory drugs (NSAID) are one of the most used drugs around the world because their effects like analgesics, antipyretic, and anti-inflammatory, in addition to other effects such as antiplatelet agents. This text aims to outline the most common side effects related to the use of NSAID. Self-medication is a common practice with multiple manifestations of adverse effects such as ulcers, infection, bleeding and intestinal perforation, hepatotoxicity, acute renal failure, among others. The development of this review was carried out in a wide search in Science Direct on the most recently published evidence, in addition to literatures with foundations relevant to this text, where it is concluded that adverse effects represent a public health problem as the mortality rate in emergency rooms across the country increases.

Key words: Drug. Therapeutic. Toxicity. Pathology.

\section{Introducción}

Los antiinflamatorios no esteroideos (AINE) son un grupo de medicamentos químicamente disímiles entre sí, que difieren en sus acciones: antipirética, analgésica y antiinflamatoria. Esta clase de fármacos incluye derivados del ácido salicílico (AAS), ácido propiónico, ácido acético, ácido enólico, fenamatos e inhibidores

\section{Correspondencia:}

*Jesús Rivera-Aguirre

E-mail: riveraaguirrejesus7@gmail.com

Disponible en internet: 30-07-2021

Fecha de recepción: 28-03-2019

Fecha de aceptación: 05-04-2021 DOI: 10.24875/REIE.19000026
Rev Educ Investig Emer. 2021;3(2):66-73 www.medicinadeemergencias.com

2604-6520 @ 2021 Sociedad Mexicana de Medicina de Emergencias, AC. Publicado por Permanyer México SA de CV. Este es un artículo open access bajo la licencia CC BY-NC-ND (http://creativecommons.org/licenses/by-nc-nd/4.0/). 
selectivos de la ciclooxigenasas (COX) 2, que actúan mediante la inhibición de las enzimas COX que catalizan el primer paso de la biosíntesis de prostanoides (Figs. 1 y 2). Estas enzimas convierten el ácido araquidónico a un compuesto intermedio inestable, la prostaglandina G2 (PGG2) y la prostaglandina GH2 (PGH2), que ocasiona la producción de prostanoides, tromboxano A2 (TXA2) y diversas protaglandinas ${ }^{1}$. Por lo tanto, al inhibir la función fisiológica de la enzima COX disminuyen las prostaglandinas, lo que conlleva efectos secundarios perjudiciales ${ }^{2}$ que se exacerban en los casos de uso crónico y toma de estos fármacos en dosis elevadas.

\section{Objetivo}

Revisar los efectos adversos más comunes relacionados con el uso de antinflamatorios no esteroideos de manera crónica, dosis elevadas y ambas posibilidades.

\section{Epidemiología}

Este tipo de medicamentos, de venta libre en el caso de EE.UU., supone el $40 \%$ del total de las medicinas consumidas, principalmente por dolor, el cual es una causa común de consulta que fue estimada en el año 2016 con un 11 al 40\% de presencia en el total de la población norteamericana en forma de dolor crónico. España sitúa la prevalencia de la automedicación alrededor de un $40-75 \%$. En un estudio realizado en Sevilla sobre el consumo de AINE, se demostró que el $48.2 \%$ del consumo total $(100 \%)$ de AINE era de consumo automedicado, lo cual fue casi tan frecuente como los que no se automedicaron y prefirieron acudir a consulta para la prescripción médica ${ }^{3,4}$. Al norte de España, en Bilbao, el AAS es el fármaco más empleado para la automedicación, seguido muy de lejos por el resto de $\mathrm{AINE}^{5}$, seguramente por su efecto cardioprotector, el cual es mal interpretado por la población en general, dándole un uso erróneo y sin fundamentos terapéuticos, ya que de todos los casos de hemorragia gastrointestinal existentes en nuestro medio, algo más de un $40 \%$ son atribuibles en conjunto al AAS y demás AINE, no siendo raro que estos pacientes tomen AAS para tratar un cuadro de dispepsia que posteriormente puede agravarse a sangrado ${ }^{6}$. Con la finalidad de evitar las complicaciones gastrointestinales de los AINE tradicionales, se diseñaron los AINE selectivos a COX-2, como el celecoxib, los cuales también manifiestan efectos adversos a nivel cardiovascular mediante la disrupción en las funciones de la prostaciclina (vasodilatador) y el tromboxano (vasoconstrictor) ${ }^{7}$.

En Pachuca-Mineral de la Reforma, en el Estado de Hidalgo, se recolectaron dos grupos de datos interpretados en ventas por kilogramo de AINE. Estos dos grupos fueron comparados clasificándolos en venta libre contra la prescripción médica. Esta comparación representa el problema de la automedicación, ya que en el resultado se observó que los cinco fármacos que se comercializan principalmente por venta libre son seleccionados de forma distinta a los seleccionados en el grupo de ventas por prescripción. En orden descendente, por venta libre: naproxeno, paracetamol, ibuprofeno, AAS y diclofenaco. Y por venta por prescripción: paracetamol, naproxeno, AAS, diclofenaco e ibuprofeno. La indicación de naproxeno es especialmente benéfica para pacientes cardiópatas, lo que conduce a la interrogante sobre si en el Estado de hidalgo la mayoría de la población son cardiópatas, o fue el naproxeno el que tuvo la mejor mercadotecnia. Añadido, a la mala indicación de estos fármacos por venta libre contra los consumidos por prescripción, los volúmenes de venta en farmacias por venta libre oscilan entre 6 y $30 \mathrm{~kg}$ al año, a diferencia del consumo por prescripción, donde se venden desde menos de un kilo hasta $22 \mathrm{~kg}$ al año, superando por mucho el consumo en el grupo de venta libre al de la prescripción médica8.

En un lapso entre 2005 y 2016 se publicaron 3,783 artículos respecto al consumo de AINE y riesgo cardiovascular, dentro de la base de datos PubMed ${ }^{9}$. Varios estudios demuestran que a partir de los 65 años la historia previa de enfermedad ulcerosa, las dosis altas de AINE, el uso concomitante de anticoagulantes, esteroides y/o ASS, y la infección por Helicobacter pylori (H. pylori) son factores determinantes de daño por AINE. El Colegio Americano de Gastroenterología indica medidas de gastroprotección de acuerdo con el riesgo de afección gastrointestinal, que se clasifica como bajo, moderado y alto, concluyendo que la medida profiláctica primaria es utilizar dosis estándar de inhibidor de la bomba de protones (IBP), así como el AINE menos ulcerogénico y a la dosis efectiva más baja. Sin embargo, a pesar de las guías, existe evidencia de un inadecuado uso ante las medidas de profilaxis primaria. La evidencia demuestra que la aplicación de medidas profilácticas reduce de forma considerable estos cuadros clínicos exacerbados, que ocasionan más de 100,000 hospitalizaciones al año en los EE.UU. y de 7,000 a 10,000 muertes anuales principalmente en pacientes clasificados como de riesgo alto ${ }^{10}$. 


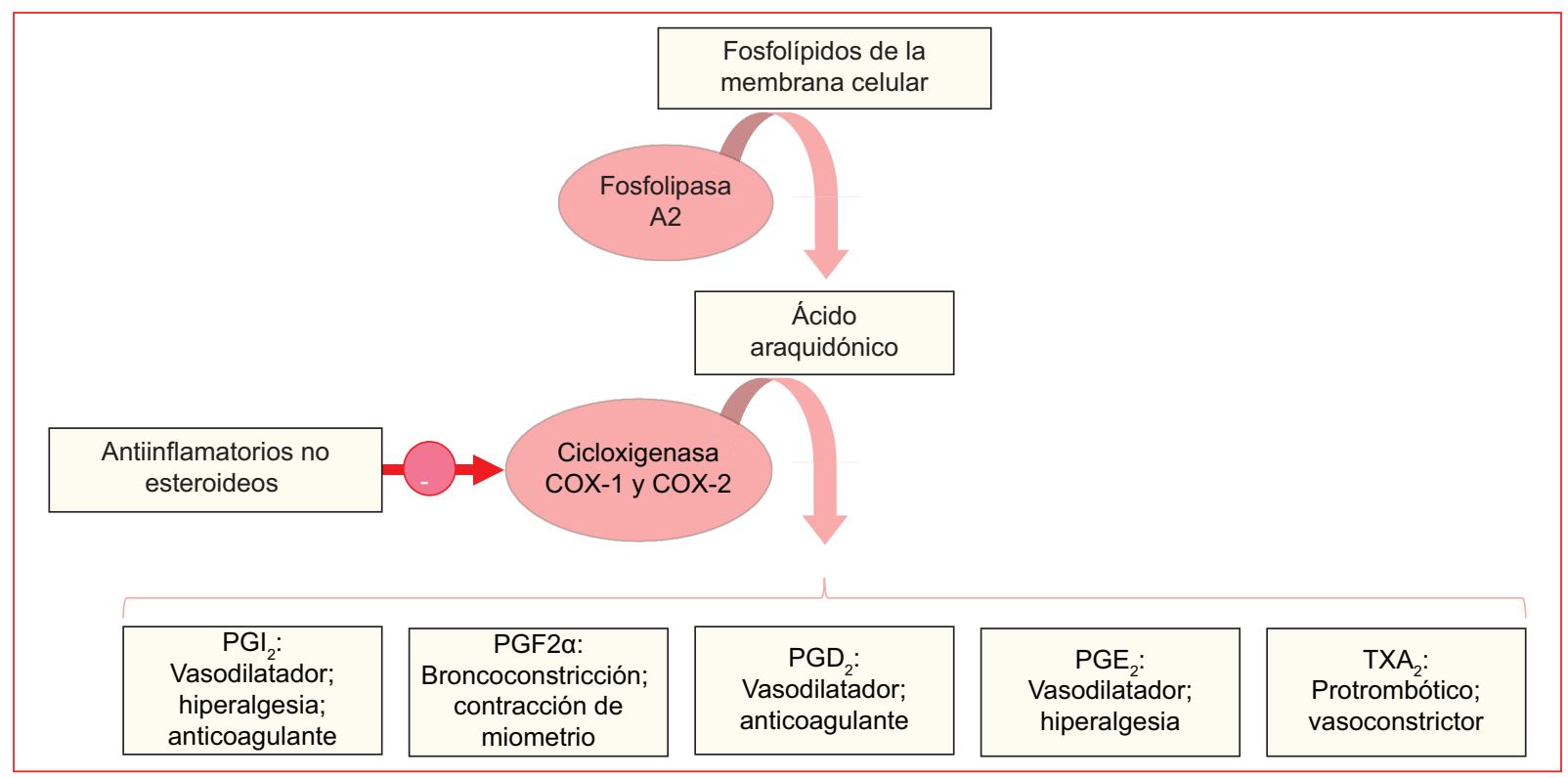

Figura 1. Inhibidores de la ciclooxigenasa. Los antiinflamatorios no esteroideos tienen la capacidad común de inhibir la enzima COX en sus dos isoformas: COX-1 y COX-2. Esta inhibición interfiere con la síntesis de PG y TX, impidiendo distintos efectos fisiológicos, como es el caso de las prostaglandinas PGI2, PGF2 $\alpha$, PGD2 y PGE2, y el tromboxano TXA2. COX: ciclooxigensa; PG: protaglandina; TX: tromboxano.

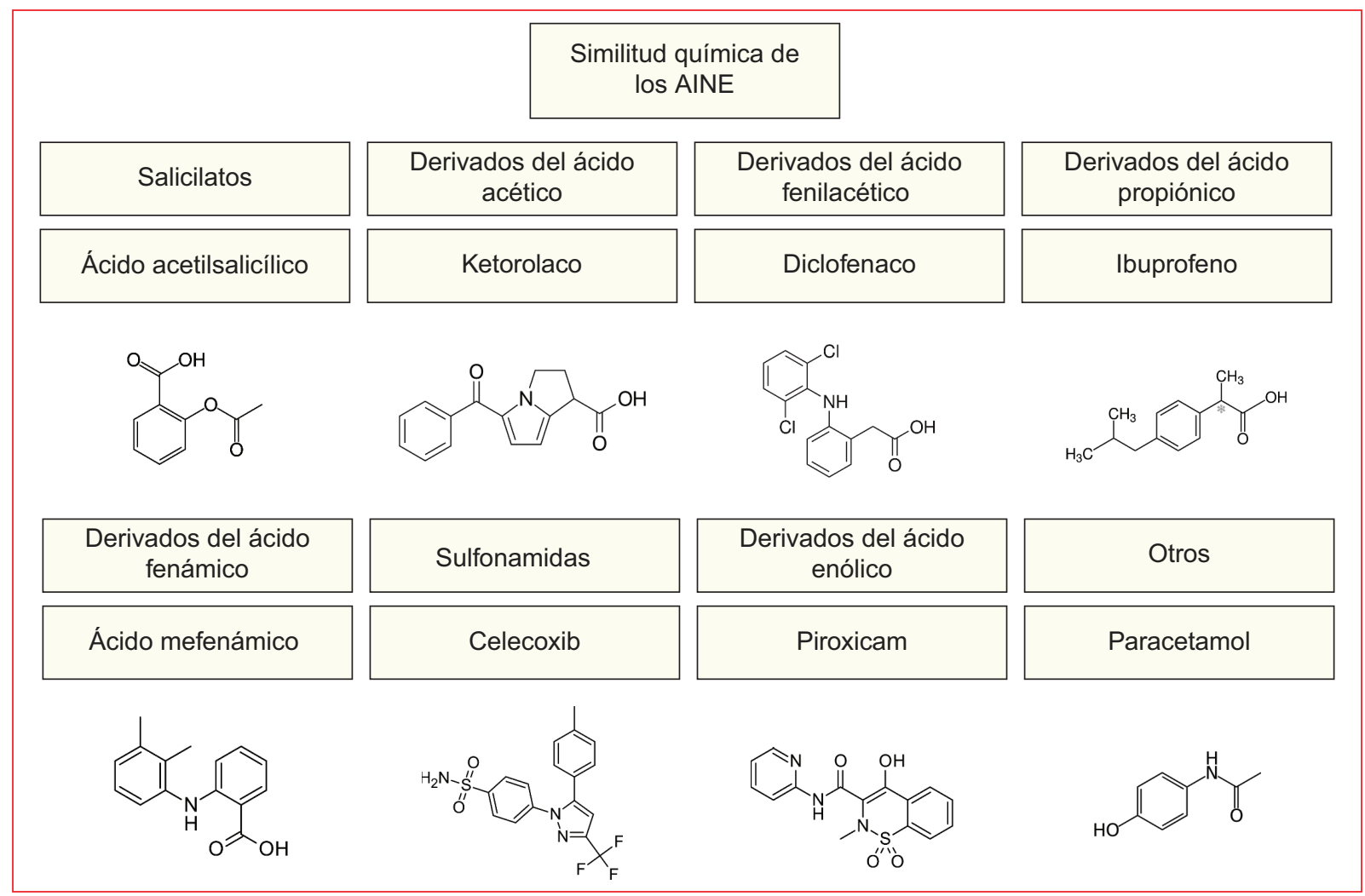

Figura 2. Similitud química de los antiinflamatorios no esteroideos. Actualmente existe una gran variedad de AINE, los cuales son clasificados en base a su similitud química en diferentes grupos, como lo son el ácido acetilsalicílico perteneciente a los salicilatos, el ketorolaco perteneciente a los derivados del ácido acético, el diclofenaco perteneciente a los derivados del ácido fenilacético, el ibuprofeno perteneciente a los derivados del ácido propiónico, el ácido mefenámico perteneciente a los derivados del ácido fenámico, el celecoxib perteneciente a las sulfonamidas, el piroxicam perteneciente a los derivados del ácido enólico y otros como el paracetamol. 
La tasa de mortalidad entre los pacientes que son hospitalizados por sangrado de tubo digestivo alto por el uso de AINE es del 5 al $10 \%{ }^{11}$. Se ha estimado que la incidencia de complicaciones gastrointestinales clínicamente relevantes entre individuos no usuarios de ASS o AINE es significativamente menor que en aquellos que sí consumen este tipo de fármacos de manera habitual. En general, la tasa de incidencia de complicaciones graves es de aproximadamente un caso por 1,000 personas por año entre los no usuarios de AINE, sin embargo, dicha tasa se incrementa en forma notable con la edad hasta 3-10 por 1,000 personas/año en pacientes mayores de 80 años, y además se presenta en el doble en varones comparada con mujeres en cada grupo de edad. Esta tasa se podría modificar por el uso de AINE y ASS que consumen las personas por autoprescripción, situación que en México es muy común, por lo que se debe tener en cuenta este posible sesgo en todos los estudios clínicos que evalúen los efectos adversos de los AINE ${ }^{12}$.

\section{Etiología}

La toma de AINE de manera contraindicada, automedicación, dosis elevadas y/o por tiempos prolongados representa la primera causa por la cual se manifestarán sus efectos adversos, lo que genera un problema para el sector salud aumentando los ingresos de hospitalización o la demanda de consulta programada, que se puede traducir en un problema de salud pública y ambiental. La decisión individual de la automedicación sin prescripción puede suponer el ahorro de tiempo y dinero que conlleva una visita médica, sin embargo, entendemos por automedicación a la administración de medicamentos sin la intervención del médico, siendo en algunas ocasiones anteriormente prescritos por el médico en circunstancias sintomáticas análogas. Otra forma de promover la automedicación ocurre con el almacenamiento de los AINE en los hogares, escuelas y sitios de trabajo, siendo una práctica muy extendida alrededor de la república mexicana llevando la ingesta de AINE a una práctica muy habitual. Se considera positivo fomentar una asesoría a los ciudadanos en todo lo relacionado con la salud, pero si no se ofrece información terapéutica adecuada sobre el uso correcto de estos fármacos puede llegar a ser perjudicial para el paciente y para las instituciones de salud pública. Por lo tanto, la automedicación es una realidad que debe aceptarse, así como también la información y la educación sanitaria han de ayudar a que se haga de forma responsable.

\section{Patogenia}

\section{Acciones sobre el aparato gastrointestinal}

La bibliografía confirma que algunos factores de riesgo son determinantes con la posibilidad de sangrado gastrointestinal en usuarios de AINE, como son: edad avanzada (mayor de 65 años), historia de episodio gastrointestinal previo (complicado o no complicado) y en pacientes que reciben terapia concomitante con esteroides y anticoagulantes. Antes de indicar un AINE, se debe evaluar cada caso individual en cuanto a factores de riesgo como edad, uso de otros agentes potencialmente agresivos al tracto gastrointestinal, tabaquismo y uso concomitante de ASS, para seleccionar la mejor opción de tratamiento para cada paciente en particular y determinar la necesidad de terapia gastroprotectora, y dada la posibilidad de riesgo cardiovascular de los COX-2 selectivos, también se deben analizar los factores de riesgo cardiovascular que tenga el paciente antes de iniciar un medicamento de este tipo, principalmente teniendo en cuenta que el efecto protector de los COX-2 selectivos disminuye considerablemente en pacientes que reciben terapia con aspirina $a^{9,10,13}$.

Los efectos adversos más frecuentes de los AINE son gastrointestinales, su uso automedicado de manera crónica causa lesiones digestivas que varían desde dispepsia, sangrado y perforación intestinal, entre otros $^{6}$. En condiciones fisiológicas, la producción de prostaciclina PGI2 inhibe la secreción de ácido gástrico y la PGE2 y PGF2 $\alpha$ estimulan la síntesis de moco protector tanto en el estómago como en el intestino delgado. Estas funciones son suprimidas con el uso de inhibidores de la COX-1 que reducen las concentraciones de estas prostaglandinas aumentando el riesgo de úlcera, sangrado y perforación intestinal².

\section{Acciones sobre el efecto antiplaquetario}

El TXA2 favorece a la agregación plaquetaria, en tanto que el PGI2 la disminuye. El AAS inhibe de forma irreversible la formación de TXA2 mediada por COX-1², logrando el efecto antiplaquetario que hace que en casos clínicos como infarto, ya sea antes o después de este, esté indicado el consumo del ASS. Esto por otra parte resulta sumamente perjudicial en pacientes que cuenten con un riesgo elevado de sangrado como úlceras gastrointestinales, que podría favorecer a la posterior complicación de estos casos clínicos, teniendo como efecto final la muerte. 


\section{Acciones sobre el riñón}

Las prostaglandinas se producen en varias localizaciones dentro del riñón, que incluyen el endotelio glomerular y vascular, las células de los túbulos colectores tubulares medulares y corticales, estos últimos en menor grado, y las células intersticiales renomedulares $^{14-16}$. Las prostaglandinas renales tienen importantes funciones locales y escasa actividad sistémica, pues se metabolizan rápidamente en la circulación pulmonar, sin embargo, la inhibición de estas a causa de AINE produce efectos adversos diversos, incluyendo disminución de la función renal, hipercalemia, hiponatremia, retención de sodio y exacerbación de la hipertensión ${ }^{17}$. El tromboxano puede causar vasoconstricción y contracción mesangial y contribuir a la disminución de la tasa de filtración glomerular y al aumento de la excreción de proteínas que frecuentemente se observan en la enfermedad glomerular ${ }^{16}$. Los efectos intrarrenales tienen importantes implicaciones clínicas debido al uso extendido de los AINE, como las acciones vasculares y tubulares en diferentes receptores de la superficie celular que pertenecen a la familia de proteínas $\mathrm{G}$ de siete receptores transmembrana ${ }^{18}$. Las prostaglandinas fundamentalmente vasodilatadoras parecen no tener ningún papel en estados basales, cuando su tasa de secreción es relativamente baja. Sin embargo, la síntesis de estas aumenta sobre todo dentro del glomérulo por vasoconstrictores como la angiotensina II, la noradrenalina, la vasopresina (ADH) (por receptores $\mathrm{V}_{1}$ ) y la endotelina. La vasodilatación posterior al efecto de las prostaglandinas contrarresta en parte la vasoconstricción neurohumoral, minimizando así el grado de isquemia renal ${ }^{19}$. El efecto clínico resultante es que los AINE no selectivos no deterioran la perfusión renal en sujetos normales, pero pueden causar isquemia renal reversible e insuficiencia renal en diversas situaciones, en particular en las patologías hipovolémicas, en las que aumentan las secreciones de angiotensina II y noradrenalina, que es el efecto compensador del cuerpo que se traduce en una mayor vasoconstricción a nivel renal. Se incluye la depleción verdadera de volumen circulante como cirrosis y la insuficiencia cardiaca. En ambos casos aumenta la gravedad de la patología subyacente con el incremento de la secreción de las tres hormonas «hipovolémicas» (angiotensina II, noradrenalina y ADH). La probabilidad de producirse insuficiencia renal aguda por AINE se relaciona con la intensidad de la vasoconstricción renal subyacente. Los pacientes con marcada retención de sodio y niveles altos de angiotensina II, noradrenalina y $A D H$ a menudo presentan una importante disminución de la tasa de filtración glomerular si se administran AINE. El efecto deletéreo suele ser revertido rápidamente al interrumpir la administración del fármaco. La actividad vasodilatadora de las prostaglandinas renales también actúa disminuyendo la resistencia vascular sistémica. Así, la administración de AINE no selectivo en pacientes hipertensos tratados con diuréticos, bloqueantes betaadrenérgicos 0 inhibidores de la enzima convertidora de angiotensina puede incrementar la tensión arterial una media de 3-5 hasta $5-10 \mathrm{mmHg}$ en algunos pacientes ${ }^{20,21}$. Dosis terapéuticas de salicilatos producen cambios definidos en el equilibrio ácido básico y en el patrón electrolítico. La compensación para el evento inicial, la alcalosis respiratoria, se logra con el incremento de la excreción renal de bicarbonato, lo que se acompaña de incremento en la excreción de $\mathrm{Na}+$ y $\mathrm{K}+$; se reducen las concentraciones plasmáticas de bicarbonato y el $\mathrm{pH}$ sanguíneo regresa a lo normal. Esta es la etapa de ácidos renal compensadora; se observa más a menudo en adultos que reciben tratamiento intensivo con salicilatos y rara vez continúa, a menos que sobrevengan efectos tóxicos. Los salicilatos causan retención de sal y agua, así como reducción aguda de la función renal en pacientes con insuficiencia cardiaca congestiva, nefropatía o hipovolemia. El uso a largo plazo de salicilatos rara vez se acompaña de nefrotoxicidad, pero la ingestión prolongada y excesiva de mezclas de analgésicos que contienen salicilatos en combinación con otros AINE puede producir necrosis papilar y nefritis intersticial, entidad clínica definida como nefropatía por analgésicos ${ }^{1,16}$.

\section{Efectos cardiovasculares}

Los inhibidores selectivos de la COX-2 se han relacionado con mayor riesgo de trastornos cardiovasculares, quizá por disminución de producción de PGI2 mediada por la COX-2. Un mayor riesgo de alteraciones cardiovasculares, incluidos infarto al miocardio y accidente cerebrovascular se han asociado con todos los AINE excepto el AAS, esto provocado por la interrupción de la homeostasis adquirida vía una prostaciclina que funge como vasodilatador, en contraste con el tromboxano, que funciona como vasoconstrictor ${ }^{22}$. La administración de AINE, exceptuando el AAS, a pacientes con enfermedad cardiovascular establecida no es recomendable. Para pacientes con enfermedad cardiovascular que no pueden evitar el uso de AINE, el naproxeno parece ser el menos lesivo ${ }^{9}$. La disminución de la síntesis de prostaglandinas induce retención de 
sodio y agua y puede causar edema en algunos pacientes. Los pacientes con antecedentes de insuficiencia cardiaca y nefropatía tienen un riesgo notablemente alto. Estos efectos también pueden mitigar los efectos de los antihipertensivos ${ }^{2,9}$. El aumento de la resistencia vascular inducido por los AINE pueden tener un efecto deletéreo adicional en pacientes con insuficiencia cardiaca avanzada. La sobrecarga que se asocia a estos casos puede causar una reducción de la contractilidad y del gasto cardiaco ${ }^{23}$. El uso de fármacos AINE está contraindicado (excepto el ASS) en los síndromes coronarios agudos, por lo que debe interrumpirse su administración. Tanto los fármacos selectivos de COX-2 como no selectivos no deben administrarse durante la hospitalización por infarto agudo al miocardio con elevación del segmento ST por el aumento del riesgo de mortalidad, reinfarto, hipertensión, insuficiencia cardiaca y ruptura miocárdica asociada con su uso 24 .

Los efectos adversos en relación cardiovascular respecto a los AINE más comunes son: el riesgo de muerte con el diclofenaco aumenta desde el principio del tratamiento y persiste a lo largo de este, además se asocia con mayor riesgo de muerte cardiovascular y muerte coronaria con una relación dependiente de la dosis; el ketorolaco presenta mayor riesgo de infarto agudo al miocardio nuevo, administrado tanto por vía oral como parenteral; el ibuprofeno aumenta el riesgo de muerte a partir de una semana de tratamiento; los inhibidores selectivos de la COX-2 incrementan el riesgo de eventos cardiovasculares desde el primer año de consumo tras un evento cardiovascular previo; el naproxeno se asocia al riesgo cardiovascular más bajo que el resto de AINE$^{9}$.

\section{Efectos adversos en pacientes asmáticos}

Los AINE son inhibidores de la COX y, por tanto, inhiben la síntesis de prostaglandinas pero no la de leucotrienos. Por esta razón los AINE deben administrarse con precaución a pacientes con asma, porque la inhibición de la síntesis de prostaglandinas puede ocasionar un cambio orientado hacia la producción de leucotrienos y en consecuencia aumentar el riesgo de exacerbaciones del asma ${ }^{1,2}$.

\section{Efectos adversos sobre el sistema nervioso central}

Es posible observar efectos adversos como cefalea, acúfenos y mareo ${ }^{2}$ en todo paciente bajo tratamiento de AINE.

\section{Efectos relacionados con hipersensibilidad}

Alrededor del $15 \%$ de los pacientes que toman AAS experimenta reacciones de hipersensibilidad. Los síntomas de alergia real incluyen urticaria, broncoconstricción y angioedema. El choque anafiláctico letal es raro. Los pacientes con hipersensibilidad grave al AAS deben evitar los AINE$^{2}$.

\section{Efectos adversos durante el embarazo y la lactancia}

La expresión de COX-2 miometrial y las concentraciones de PGD2 y PGF2 $\alpha$ incrementan en forma notable en el miometrio durante el trabajo de parto. Se ha demostrado en seres humanos la prolongación de la gestación con la administración de AINE. Algunos AINE, en particular la indometacina, se han utilizado para interrumpir el trabajo de parto prematuro, aunque esta indicación no ha sido autorizada. Sin embargo, su uso de esta manera se acompaña de cierre del conducto arterioso y afectación de la circulación fetal in utero, en particular en fetos de más de 32 semanas de gestación. Los inhibidores selectivos de la COX-2 se han utilizado como fármacos tocolíticos; este uso se ha asociado con estenosis del conducto arterioso y oligohidramnios. Por último, el uso de AINE y AAS al final del embarazo puede incrementar el riesgo de hemorragia puerperal. Por tanto, el embarazo, en especial cerca de su término, es una contraindicación relativa para el uso de todos los AINE. Además, su uso debe compararse con los riesgos potenciales para el feto, incluso en casos de trabajo de parto prematuro y, en especial, en casos de hipertensión inducida por el embarazo ${ }^{1,2}$.

\section{Diagnóstico}

El clínico debe ser muy analítico de lo que se prescribe, además de lo que no es prescrito, como lo son Ios AINE, para prevenir comorbilidades, así como también la toma inadecuada de decisiones pueden empeorar dichas comorbilidades.

Un cuadro agudo o crónico deberá ser diagnosticado mediante la buena historia clínica, con especial énfasis sobre la condición o cuadro clínico adyacente responsable que fuerza al paciente a automedicarse, usar AINE de forma crónica o en dosis muy altas, combinación de estos, y teniendo claras las interacciones farmacológicas de cada paciente. 


\section{Metodología}

La elaboración de esta revisión se realizó en una amplia búsqueda en Science Direct sobre la evidencia más recientemente publicada con no más de cinco años de antigüedad, utilizando las palabras clave: fármaco, terapéutica, toxicidad, patología. Eligiendo idioma español e inglés, con preferencia en los textos de origen centroamericano. Además de bibliografías con fundamentos importantes para el desarrollo de esta investigación.

\section{Conclusión}

La vigilancia en el consumo de los AINE es una guía útil para el tratamiento, pero debe utilizarse en combinación con la valoración del estado clínico general del paciente, tiempo transcurrido desde el consumo y la dosis. No existe un antídoto específico para la amplia gama de efectos adversos del AINE. El tratamiento inicia con un buen interrogatorio clínico, aplicando una terapéutica sintomatológica y corrigiendo los efectos adversos exacerbados.

Factores clave para prevenir en lo posible los efectos adversos y contraindicaciones de los AINE son:

- El diferente grado de selectividad que tenga el fármaco para la inhibición entre la enzima COX-1 y la COX-2, como, por ejemplo, el meloxicam y el etodolaco (preferencialmente inhibidores de la COX-2), que no llegan a ser inhibidores selectivos, como lo son el celecoxib o el etoricoxib. De manera similar sucede en los AINE más selectivos para COX-1 como el ibuprofeno, el ketoprofeno y la indometacina ${ }^{25}$.

- El segundo factor clave es el potencial riesgo que tiene el fármaco de provocar interacciones con otros fármacos; por ejemplo, el ASS tiene una relación en el aumento de riesgo cardiovascular en interacción con inhibidores selectivos de la COX-2. Además, combinar el ASS a dosis menores de $100 \mathrm{mg}$ con cualquier otro AINE puede interferir con los efectos antiplaquetarios 26,27 .

Comparando a los inhibidores de la COX tradicionales contra los inhibidores selectivos de la COX-2, existen diferencias significativas entre ellos. El diclofenaco a dosis de $150 \mathrm{mg}$ por día provoca un aumento en infarto al miocardio por aumento de eventos vasculares, en comparación de otros fármacos como el naproxeno y el ibuprofeno, el diclofenaco tiene perfil de riesgo más alto de muerte cardiovascular ${ }^{28}$. El ibuprofeno (2,400 mg por día) se asocia a casos de eventos coronarios. Por el contrario, no hay un aumento ni de eventos vasculares ni de eventos coronarios, en pacientes que consumen altas dosis de naproxeno a $500 \mathrm{mg}$ dos veces al día. El riesgo de insuficiencia cardiaca aumenta el doble en el caso de todos los AINE sin excepción ${ }^{29}$. El ASS es aceptable en casos de prevención primaria y secundaria de enfermedad cardio-cerebrovascular ${ }^{30}$. El celecoxib, con una inhibición de COX-1 de tan solo un 10\% a dosis terapéuticas, no presenta alguna característica clínicamente relevante sobre la COX-131. Respecto al efecto antiplaquetario del ASS, con $81 \mathrm{mg}$ por día no se interfiere su acción cardioprotectora cuando es añadido ibuprofeno de $200 \mathrm{mg}$ después de dos horas de la dosis de ASS $^{32}$. Por lo que los AINE deberían ser cautelosamente usados en pacientes que requieren ASS para cardioprotección ofreciendo buenas alternativas como terapias tópicas (los efectos adversos siempre son menores si se aplica de forma tópica). El uso simultáneo de AINE y un IBP puede disminuir el riesgo de úlcera, pero este efecto no protege zonas posteriores al duodeno ${ }^{33,34}$. Es importante considerar que en pacientes que a pesar de la prevención de efectos adversos gastrointestinales continúan teniéndolos, debería optarse por alguna alternativa del tratamiento de AINE tradicional, como un IBP y un fármaco selectivo como celecoxib o preferente a COX-2 como el meloxicam, para pacientes con moderado 0 alto riesgo de efectos gastrointestinales. A pacientes bajo tratamiento con AINE siempre se debe aconsejar nunca ingerir el medicamento con el estómago vacío. En pacientes en los cuales se encuentra presente tanto enfermedad cardiovascular como enfermedad gastrointestinal, o múltiples factores de riesgo, el tratamiento con AINE debería ser revisado minuciosamente con cada inspección médica.

Los AINE pueden provocar retención de líquido, edema y ultimadamente hipertensión, daño agudo renal, necrosis tubular, necrosis papilar e insuficiencia renal. Esto incrementa en pacientes que mantienen tratamiento con inhibidores de la enzima convertidora de angiotensina con o sin diurético añadido, ya que la eficacia de estos medicamentos puede atenuarse por Ios AINE debido a la inhibición de la vasodilatación ${ }^{35}$. Todos los AINE deberían ser evadidos en todo paciente con enfermedad renal avanzada.

La población suele ingerir a dosis y de manera inadecuada estos fármacos por su fácil acceso, lo que produce un problema de salud pública, al aumentar el índice de mortalidad en las salas de emergencia de todo el país. 


\section{Financiamiento}

La presente investigación no ha recibido ninguna beca específica de agencias de los sectores público, comercial o sin ánimo de lucro.

\section{Conflicto de intereses}

El autor declara no tener conflicto de intereses.

\section{Responsabilidades éticas}

Protección de personas y animales. Los autores declaran que para esta investigación no se han realizado experimentos en seres humanos ni en animales.

Confidencialidad de los datos. Los autores declaran que en este artículo no aparecen datos de pacientes.

Derecho a la privacidad y consentimiento informado. Los autores declaran que en este artículo no aparecen datos de pacientes.

\section{Bibliografía}

1. Hilal-Dandan R, Brunton LL. Las bases farmacológicas de la terapéutica. 12. ${ }^{\mathrm{a}}$ ed. McGraw Hill Education; 2015.

2. Whalen K. Farmacología. 6. ${ }^{\underline{a}}$ ed. Barcelona: Wolters Kluwer; 2016.

3. Darr U, Sussman NL. Drug-induced liver injury in the setting of analgesic use. Clin Liver Dis. 2019;1089:3261-19.

4. Montaño A, Torelló J, Castillo JR, Cayuela A, Moreno I, Fernandez I. Conocimientos y actitud de los usuarios en relación al empleo de medicamentos para el dolor, la fiebre o la inflamación. Aten Primaria. 1994;13(4):165-70.

5. González-Amayuelas M, Mendibil-Crespo I, Gutiérrez-Martínez I, Blanco-Alonso MÁ. Automedicación con AINE por los usuarios de dos consultas de Atención Primaria. Gac Med Bilbao. 2006;103:54-7.

6. Hawkey CJ. Non-steroidal anti-inflammatory drug gastropathy: Causes and treatment. Scand J Gastroenterol. 1996;220:124-7.

7. Hatt KM, Vijapura A, Maitin I, Cruz E. Safety considerations in prescription of NSAIDs for musculoskeletal pain: A narrative review. PM R. 2018;10(12):1404-11.

8. Rodríguez-Anaya A, Octavio-Aguilar P, Gaytán-Oyarzún JC. Consumo de fármacos antiinflamatorios no esteroideos en Pachuca, Hidalgo. Laboratorio de Genética, Centro de Investigaciones Biológicas, Universidad Autónoma del Estado de Hidalgo. Salud Publica Mex. 2015;57(5):365-6.

9. Muñoz Olmo L, Juan Armas J, Gomariz García JJ. Riesgo de eventos fatales/no fatales en pacientes con enfermedad coronaria/infarto agudo de miocardio previo y tratamiento con antiinflamatorios no esteroideos. SEMERGEN. 2017;44:355-63.

10. Velasco-Zamora JA, Gómez-Reyes E, Uscanga L. ¿Qué tanto se siguen las recomendaciones de las guías clínicas sobre gastroprotección? Una revisión en enfermos que consumen antiinflamatorios no esteroideos. Rev Gastroenterol Méx. 2016;81(3):119-20.

11. Armstrong CP, Blower AL. Non-steroidal anti-inflammatory drugs and life threatening complications of peptic ulceration. Gut. 1987;28:527-32.
12. Hernández-Díaz S, Rodríguez LA. Incidence of serious upper gastrointestinal bleeding/perforation in the general population: review of epidemiologic studies. J Clin Epidemiol. 2002;55:157-63.

13. Caamaño $F$, Figueras $A$, Lado $E$, Gestal-Otero JJ. La automedicación: concepto y perfil de sus "usuarios". Gac Sanit. 2000;14(4):294-9.

14. Bonvalet JP, Pradelles P, Farman N. Segmental synthesis and actions of prostaglandins along the nephron. Am J Physiol. 1987;253:F377.

15. Farman N, Fradelles P, Bonvalet JP. PGE2, PGE2 $\alpha, 6$-keto-PGF1 $\alpha$ and TXB2 synthesis along the rabbit nephron. Am J Physiol. 1987;252:F53-9.

16. Remuzzi F, Fitzgerald GA, Patrono $C$. Tromboxane synthesis and action within the kidney. Kidney. 1992;41:1483.

17. Clivea DM, Clive PH. Nonsteroidal antiinflammatory drugs and opioids in chronic kidney disease. En: Kimmel P, Rosenberg M, editores. Chronic Renal Disease. Second Edition. Elsevier; 2020.

18. Breyer MD, Jacobson HR, Breyer RM. Functional and molecular aspects of renal prostaglandin receptors. J Am Soc Nephrol. 1996;7:8

19. Rose BD, Post TW. Clinical physiology of acid-base and electrolyte disorders. $6^{\text {th }}$ edition. McGrawHill; 2020.

20. Pope JE, Anderson JJ, Felson DT. A meta-analysis of the effects of non-steroidal anti-inflamatory drugs on blood preassure. Arch Intern Med. 1993;153:477.

21. Sahloul MZ, al-Kiek R, Ivanovich P, Mujais SK. Nonesteroidal anti-inflamatory drugs and anti-hypertensives. Cooperative Malfeasance. Nephron. 1990;56:343.

22. Perazella MA. COX-2 selective inhibitors: analysis of the renal effects. Expert Opin Drug Saf. 2002;1:53.

23. Dzau VJ. Renal and circulatory mechanisms in congestive heat failure. Kidney Int. 1987;31:1402.

24. Sinz, E., Navarro, K., \& Soderberg, E. Advanced Cardiovascular Life Support: Provider Manual American Heart Association. American Heart Association, Estados Unidos, 2011.

25. Cryer B, Feldman M. Cyclooxygenase-1 and cyclooxygenase-2 selectivity of widely used non-steroidal anti-inflammatory drugs. Am J Med. 1998;104:413-21.

26. MacDonald TM, Wei L. Effect of ibuprofen on cardioprotective effect of aspirin. Lancet 2003;361:573-4.

27. Catella-Lawson F, Reilly MP, Kapoor SC, Cucchiara AJ, DeMarco S, Tournier B, et al. Cyclooxygenase inhibitors and the antiplatelet effects of aspirin. N Eng J Med 2001; 345:1809-1817.

28. Trelle S, Reichenbach S, Wandel S, Hildebrand P, Tschannen B, Villiger PM, et al. Cardiovascular safety of non-steroidal anti-inflammatory drugs: Network meta-analysis. BMJ. 2011;342:c7086.

29. Coxib and Traditional NSAID Trialists' Collaboration. Vascular and upper gastrointestinal effects of non-steroidal anti-inflammatory drugs: meta-analyses of individual participant data from randomized trials. Lancet. 2013;382:769-79.

30. Guirguis-Blake JM, Evans CV, Senger CA, O'Connor EA, Whitlock EP. Aspirin for the primary prevention of cardiovascular events: A systematic evidence review for the U.S. preventive services task force. Ann Intern Med. 2016;164:804-13.

31. Rowcliffe M, Nezami B, Westphal ES, Rainka M, Janda M, Bates V, et al. Topical diclofenac does not affect the antiplatelet properties of aspirin as compared to the intermediate effects of oral diclofenac: A prospective, randomized, complete crossover study. J Clin Pharmacol. 2016;56:422-80.

32. Awa $\mathrm{K}$, Satoh $\mathrm{H}$, Hori $\mathrm{S}$, Sawada $\mathrm{Y}$. Prediction of time-dependent interaction of aspirin with ibuprofen using a pharmacokinetic pharmacodynamic model. J Clin Pharm Ther. 2012;37:469-74.

33. Goldstein JL, Johanson JF, Hawkey CJ, Suchower LJ, Brown KA. Clinical trial: healing of NSAID-associated gastric ulcers in patients continuing NSAID therapydA randomized study comparing ranitidine with esomeprazole. Aliment Pharmacol Ther. 2007;26:1101.

34. Chan FK, Wong VW, Suen BY, Wu JC, Ching JY, Hung LC, et al. Combination of a cyclooxygenase-2 inhibitor and a proton-pump inhibitor for prevention of recurrent ulcer bleeding in patients at very high risk: A double randomized trial. Lancet. 2007;369:1621.

35. Varga Z, Sabzwari S, Vargova V. Cardiovascular risk of nonsteroidal anti-inflammatory drugs: An under-recognized public health issue. Cureus. 2017:9:e1144. 